\title{
Effect of Digital Lifestyle Management on Metabolic Control and Quality of Life in Patients with Well-Controlled Type 2 Diabetes
}

\author{
Chinmay Dwibedi · Birgitta Abrahamsson • Anders H. Rosengren (D)
}

Received: December 30, 2021 / Accepted: January 27, 2022 / Published online: February 12, 2022

(C) The Author(s) 2022

\begin{abstract}
Introduction: The lack of effective, scalable solutions for lifestyle treatment is a global clinical problem, causing severe morbidity and mortality. Digital tools could enable broad utility, but long-term metabolic outcomes and the influence on quality of life are unclear.

Methods: We developed a new method for lifestyle treatment that promotes self-reflection and iterative behavioural change, provided as a digital tool, and evaluated its effect on glycaemic control in patients with type 2 diabetes with $\mathrm{HbA} 1 \mathrm{c}$ below $52 \mathrm{mmol} / \mathrm{mol}(n=297)$. As a secondary analysis, its effect on quality of life (using SF-12) was examined in both participants with and without diabetes (total $n=1914$ ). The tool was evaluated during a 12-week randomization period to assess the existence of effect,
\end{abstract}

Supplementary Information The online version contains supplementary material available at https:// doi.org/10.1007/s13300-022-01214-2.

C. Dwibedi · B. Abrahamsson · A. H. Rosengren ( $\square)$ Department of Neuroscience and Physiology,

Sahlgrenska Academy at the University of

Gothenburg, Medicinaregatan 11, 40530

Gothenburg, Sweden

e-mail: anders.rosengren@gu.se

C. Dwibedi · B. Abrahamsson · A. H. Rosengren Wallenberg Centre for Molecular and Translational Medicine, University of Gothenburg, Gothenburg, Sweden with a subsequent open-label follow-up to study long-term outcomes.

Results: Participants were randomized to wait or access the intervention tool. The mean difference in $\mathrm{HbA} 1 \mathrm{c}$ was $2 \mathrm{mmol} / \mathrm{mol}(95 \% \mathrm{CI}-4$ to $0 ; P=0.02$ ) after 12 weeks in participants with type 2 diabetes. The groups were then merged to enable all participants to use the tool. The mean HbA1c reduction from baseline in patients with type 2 diabetes using the tool was $2 \mathrm{mmol} / \mathrm{mol}$ compared with matched controls (95\% CI -3 to $0 ; P=0.005)$. In users with HbA1c above $45 \mathrm{mmol} / \mathrm{mol}$, the mean difference between the groups was $4 \mathrm{mmol} / \mathrm{mol}$ (95\% CI -7 to -2$)$. The improvements were sustained during the follow-up of 1 year on average. Users of the tool also had improved quality of life from baseline to 6 months, mainly observed in non-diabetic participants.

Conclusion: The tool does not require in-person reinforcement or increased healthcare resources, and the marginal cost is fundamentally lower than pharmacological treatment and most existing lifestyle interventions. The results therefore open a new means for self-managed lifestyle treatment with long-term metabolic efficacy that can benefit large numbers of people.

Trial Registration: ClinicalTrials.gov NCT04 624321 and NCT05006508. 
Keywords: Diabetes self-management; Digital device; Lifestyle intervention; Glucose control; Patient care; Quality of life; Type 2 diabetes

\section{Key Summary Points}

Why carry out this study?

Lifestyle treatment is currently limited by the associated need for large healthcare resources, and there is a severe clinical need for low-cost patient-centric solutions with sustained efficacy.

We developed a new digital tool with very low marginal cost per patient to be used without the need for in-person reinforcement or increased healthcare activities and evaluated its effect on glycaemic control and quality of life.

\section{What was learned from the study?}

Participants using the tool had improved metabolic control compared with randomized controls during 12 weeks and compared with matched controls during a follow-up of 1 year on average.

The tool was also shown to improve physical and mental aspects of quality of life, especially in participants without diabetes.

The tool was provided as a stand-alone support under conditions that were as similar as possible to routine clinical conditions, which may reduce adherence compared with stricter regimes but increases the general significance of the results by demonstrating what can be expected in real-life situations over extended time.

\section{INTRODUCTION}

Lifestyle-related diseases such as type 2 diabetes (T2D) are major causes of death and disability

[1]. Structured lifestyle treatment is currently limited by the associated costs and need for large healthcare resources. Digital tools could potentially complement drug treatment and traditional lifestyle programmes, but most solutions require intensified healthcare activities or user fees, which preclude broad application. In light of the increasing number of afflicted individuals, it is necessary to find new means to support lifestyle self-management that can meet individual preferences in content and timing and reach many patients at low cost [2-4]. This is, however, currently hampered by several important knowledge gaps [5].

First, data on long-term effects ( $>6$ months) are scarce [5-8], and recent meta-analyses have shown declining metabolic response to digital lifestyle interventions after 6 months [5-11]. Second, digital tools are often combined with coaching or intensified healthcare contacts, making it difficult to specifically assess the effect of the digital component and apply the results to a broad range of settings [5, 8-11]. In fact, one of the major limitations to structured lifestyle support, whether traditional or digital, is that it currently requires increased efforts by healthcare professionals or costs for the individual patient, and less than one out of ten individuals with diabetes take part in such programmes [3]. Third, it is unclear whether tools aimed at managing metabolic control can also improve quality of life and be applicable to broader population groups, including individuals without diabetes $[5,9,11-13]$.

Here we evaluated a new self-managed lifestyle tool that is scalable and does not require additional healthcare resources. We hypothesized that glycated haemoglobin (HbA1c), reflecting long-term blood glucose, would decrease in patients with T2D exposed to the tool, both compared to randomized wait list controls and matched controls. We also aimed to assess the effects of the tool on quality of life in both individuals with diabetes and without diabetes. 


\section{METHODS}

\section{Overall Study Description}

We developed a new lifestyle treatment on the theoretical foundation of self-affirmation [14] and implemented it as a digital tool to enable broad applicability. The tool is web-based and is used as a stand-alone continuous support without requirement of additional healthcare activities. It is available in English and Swedish and is provided for free via academic institutions.

We conducted a clinical study (approved by the regional ethics review committee of Gothenburg, Sweden; no. 651/16, approved on 22 August 2016, and subsequent amendments) to test the hypothesis that glucose control would improve in patients with T2D using the digital tool. As a secondary analysis, we investigated its effect on quality of life in both participants with diabetes and those without. At study start, participants were randomized to access the tool or wait for 12 weeks (4:1 ratio). The groups were then merged to enable all participants to use the tool during an extended open-label period.

The study aimed to investigate metabolic outcomes and quality of life under conditions that were as similar as possible to real-life settings. After an initial screening visit, participants did not attend any further study visits but were followed at their primary care unit with analysis of metabolic control according to clinical routine. Participants also completed questionnaires on quality of life. In case of technical questions, they could contact a study coordinator, who also responded to requests to clarify content in a general manner without providing personal advice. The study was structured to assess (1) comparisons from baseline to 12 weeks between participants randomized to a control group or to access the tool; (2) openlabel comparisons from baseline to end of follow-up between users of the tool and matched controls; and (3) changes in quality of life in both participants with and without diabetes.

The study was performed according to the Declaration of Helsinki and later amendments.
All participants provided written informed consent.

\section{Enrolment and Study Procedures for Metabolic Assessments}

Patients older than 35 years in the Scania region (southern Sweden) with prior documentation of T2D were invited to screening visits at Scania University Hospital, Malmö, Sweden. Diabetes mellitus was diagnosed in routine healthcare on the basis of the World Health Organization (WHO) criteria (fasting plasma glucose $\geq 7.0 \mathrm{mmol} / \mathrm{l}$ or 2 -h post-load plasma glucose $\geq 11.1 \mathrm{mmol} / \mathrm{l}$ or $\mathrm{HbA} 1 \mathrm{C} \geq 48 \mathrm{mmol} / \mathrm{mol}$ ). Exclusion criteria for the glycaemic assessments were type 1 diabetes, maturity-onset diabetes of the young, secondary diabetes, other conditions, treatments or participation in clinical studies that in the judgement of the investigator could affect the evaluation, or conflicts of interest (such as association with the study team, funders, authorities, universities or other public or private bodies).

Participants were recruited via letters sent to patients with a known history of type 2 diabetes in the ANDIS (All New Diabetics in Scania) registry or by advertisements. Individuals who were interested in taking part contacted the study personnel via telephone, regular post, email or through a dedicated web page and were invited to a screening visit for analysis of HbA1c. A few participants had difficulties attending the university hospital because of travel issues and instead had their initial screening of HbA1c at a primary care unit. Patients with poor glycaemic control (HbA1c $\geq 52 \mathrm{mmol} / \mathrm{mol}$; standard glycaemic treatment target according to current guidelines [1]) were invited to further physical visits (as described in NCT04624321), the results of which will be reported elsewhere. Patients with HbA1c $<52 \mathrm{mmol} / \mathrm{mol}$ were included in the present study and followed prospectively with clinical data (as described in NCT05006508).

Participants received travel reimbursement for the screening visit. No other financial incentives were provided. Participants were managed by their ordinary healthcare providers 
throughout the study. They received usual care according to routine diabetes management and treatment based on the general guidelines from the European Association for the Study of Diabetes and the American Diabetes Association [1]. The open-label assessments include, however, only participants who did not change antihyperglycaemic medication during the follow-up period in order to evaluate the effect of the tool in addition to stable pharmacological treatment conditions.

Healthcare providers and study personnel were not involved in the participants' activities on the tool. The participants used it at their preferred pace but received written recommendations within the tool to complete a theme at least every other week. The recommended frequency of use intended to enable sufficient time to implement changes between sessions while maintaining awareness of the questions and being exposed to the different areas covered by the tool. Participants received regular emails to prompt continuous usage but no reinforcement by study personnel, as we wanted to assess the natural usage pattern and resultant outcomes that can be expected in real-life situations over extended time without the need for increased healthcare support.

\section{Enrolment and Study Procedures for Assessments of Quality of Life}

In addition to the study participants with T2D in the Scania region, participants were enrolled from across Sweden for assessments of quality of life. Individuals above 35 years of age with or without T2D were eligible to take part in these assessments. Participants were recruited via advertisements. Individuals who were interested in taking part contacted the study personnel via telephone, regular post, email or through a dedicated web page. They received a letter with detailed study information and a form for written informed consent, which was sent to the study centre in case they wanted to participate.

\section{Randomization Procedures}

After formal inclusion, either at a screening visit (patients with T2D in the Scania region) or following the submission of a written informed consent (participants across Sweden with or without T2D), participants received an introductory email with a secure web link to a personal study account and instructions on how to get started. By clicking the link, they set a password, completed a baseline questionnaire, and were randomized via a web-based system to have immediate access to the tool or wait for 12 weeks. In this manner, allocation was concealed to both participants and study personnel at inclusion (whether participants were eventually assigned to the tool or usual care was, however, open-label).

Participants randomized to wait received usual care via their ordinary healthcare provider. After 12 weeks they received an email message with a link to complete the questionnaire once again and were then able to access the tool.

The randomization sequence (block size of eight, unknown to study personnel) was generated by external statisticians, who had no contact with participants or study personnel. It was uploaded by external technical staff to the web-based allocation system. Thus, the generation of the random sequence, participant enrolment by study personnel, and the webbased system for allocation to randomization groups were clearly separated.

\section{Selection of Matched Controls}

After the initial 12-week period, all study participants had access to the tool during an extended follow-up. The development of glucose control in participants with T2D in the Scania region was compared with matched controls on usual care. Controls were selected from patients with T2D in the ANDIS registry. ANDIS was approved by the regional ethics review committee (584/2006 and 676/2012) and aims to register all incident cases of diabetes in Scania, which is one of the largest regions in Sweden with 1,200,000 inhabitants in both 
rural and urban areas and a wide distribution of socioeconomic background. Approximately 26,000 patients with diabetes (more than $90 \%$ of the estimated number of eligible cases in the region) are included as of December 2021. Prospective data on metabolic variables and medication are obtained via the Swedish National Diabetes Registry and clinical registries on drug prescription and laboratory tests.

The controls were matched 1:1 with study participants on gender, age (at the index date), body mass index (BMI) and HbA1c. The index date was selected at random among all registrations meeting the requirements for available follow-up time (6-18 months within which the HbA1c measurement nearest 365 days after the index date was selected) with no medication change during follow-up.

\section{The Intervention Tool}

We developed a digital tool on the theoretical foundations of self-affirmation and motivational interviewing [13, 14]. Self-affirmation theory postulates that perceived threats to one domain (e.g. sedentary behaviour) can be managed more effectively by reflecting on strengths in other domains. From that broader perspective people may regard changes to specific behaviours as less threatening to overall self-integrity, leading to a less defensive attitude. Self-affirmation is associated with both greater intentions for behavioural change and actual behavioural change [15-17]. The principles of motivational interviewing (MI) were incorporated in the tool to promote reflection on ambivalence and commitment to change, which has been shown to increase intrinsic motivation [18]. To enable this without a traditional interviewer, the digital tool contains a large number of questions to stimulate self-reflection. Self-reflection may support autonomy [12] and has played an important role in, for example, later forms of cognitive behavioural therapy [19]. It has, however, not been of major focus in diabetes self-management.

The detailed development of the tool will be described elsewhere. In brief, the tool was developed in an iterative manner with repeated testing by patients, including feedback on usability and content. Based on the feedback and observations of user behaviour, a complete digital tool was developed (in Drupal, version 7). The tool is web-based with a responsive design to enable patients to use it via their own computer, tablet or mobile phone. All data are transferred via secure protocols and stored on secure servers.

The tool is composed of 80 different themes, focusing on diet and exercise but also a range of other areas, including stress management, decision-making, social interactions, loneliness, and negative automatic thoughts. Previous systematic reviews have highlighted the importance of including defined behaviour change techniques (BCT) [5]. We therefore based the themes on the comprehensive theoretical framework and taxonomy proposed by Michie and colleagues [20], which has been widely accepted [5]. Each theme of the tool incorporates 2-4 BCT (24 BCT are used altogether). Table $S 1$ in the Supplementary Material describes the different BCT, and Table S2 presents the full list of themes in the tool.

A theme takes approximately 10-30 min to complete and contains one or more of the following components:

- A self-assessment test with automated feedback, aiming to raise awareness of current behaviour.

- An exercise such as evaluating short- and long-term benefits and losses associated with health-related goals, in order to identify willingness and barriers to change. Other exercises may involve time prioritization, mindful eating, and methods to cope with distress and automatic thoughts.

- Informative texts (approximately 500-800 words per text) on topics of relevance to health and lifestyle-related diseases. Texts are based on international consensus recommendations for lifestyle management $[1,21]$.

The various themes allow users to see how different areas are connected and how problems in one domain, e.g. unhealthy eating, could be managed by changes in other domains, e.g. stress coping. As the initial development process identified a need to frame lifestyle 
management within a larger perspective, we also include a set of themes covering the different aspects of existential health that the WHO has proposed [22-24]. These themes aim to stimulate questions on overall life context and how it relates to current habits and disease coping (Table S2).

At each login, users select a theme they consider to be of relevance. At the end of the theme, they ask a question to themselves to promote reflection on how the information and insights from the theme could be implemented in daily life. This methodology is in line with self-affirmation theory and MI [14, 18]. It aims to facilitate sustainable lifestyle changes by raising general awareness of current priorities and helping patients explore different options and choose their own course of action.

The tool is supposed to be used as a continuous support without a finite number of sessions, and users are encouraged to return to the tool within 2 weeks and are then given the opportunity to comment briefly in a diary on whether they have done any behavioural changes. They select a new theme or revisit one they have previously found valuable. Users can refine earlier questions or ask new ones, in order to stimulate continuous reflection as they proceed through the tool.

The tool is maintained and provided via academic institutions (University of Gothenburg, Sweden). It is technically prepared for multiple languages with a language select function and is currently available in English and Swedish. The yearly expenses related to the tool consist of server cost ( $€ 0.12$ per user), email expenses ( $€ 0.21$ per user), as well as administration and technical personnel for user requests, continuous updates and improvements to the tool ( $€ 6.34$ per user). These numbers are based on current actual costs for providing the service to 50,000 users (including the participants in the present and other ongoing studies).

\section{Clinical Study Outcomes}

The primary study variable was HbA1c in blood. Screening values were obtained at the study centre or at primary care units, and subsequent outcome data were obtained from routine clinical measurements of HbA1c in primary care.

The short form-12 (SF-12) health survey was used to assess quality of life. It was completed at baseline and after the 3-month randomization period as well as 6 months after accessing the tool. The distinct components of the survey can be summarized as a physical health score and a mental health score, with higher values implying better health [25]. The SF-12 questionnaire was separate from the tool and completed by participants via their study account online using a reminder system including emails that were sent when a new questionnaire was to be completed. Participants were instructed to complete the questionnaire on their own to avoid observer bias.

\section{Baseline Data}

The following baseline data were collected electronically before completing the first questionnaire: age, sex, BMI, prevalence of T2D, time since diabetes diagnosis, known diabetic complications (retinopathy, neuropathy, nephropathy and diabetic foot disease) and current glucose-lowering treatment in case they had T2D, as well as comorbidities. At quarterly intervals during follow-up, the participants with T2D were requested to report any changes in glucose-lowering medication.

\section{Statistics}

\section{Primary endpoints}

The two primary endpoints were:

- The change of HbA1c relative to baseline compared between participants randomized to wait and randomized to access the tool. Comparisons between randomized controls and participants with immediate access to the tool were performed for participants with T2D in the Scania region who had an HbA1c measurement obtained in routine care within 30-180 days after study inclusion, utilizing the measurement nearest to 90 days after inclusion. Participants were 
included in the analysis independent of frequency of using the tool according to the intention to treat principle. No imputation was done for missing values in patients who lacked clinical data within the time window. The change of HbA1c relative to baseline at inclusion was compared between randomization groups using a two-sided independent $t$ test. We needed 90 participants in the intervention group and 23 in the control group to ensure at least $80 \%$ power at alpha $=0.05$ to detect a significant difference between the groups, assuming that the true treatment effect of the tool is $2 \mathrm{mmol} / \mathrm{mol}$ with a standard deviation of $3 \mathrm{mmol} / \mathrm{mol}$ for the change of HbA1c. (The standard deviation for the change of HbA1c during 12 weeks was estimated from patients in the ANDIS cohort with baseline HbA1c $<52 \mathrm{mmol} / \mathrm{mol}$.)

- The change of HbA1c from baseline to end of follow-up in participants using the tool compared to matched controls.

For analysis from baseline to end of followup, the baseline HbA1c at inclusion and the HbA1c measurement obtained in clinical care nearest to 365 days after inclusion within a window of 18 months after inclusion were used. The HbA1c data were obtained via clinical data, which were made available continuously for ANDIS patients. The reported follow-up time in the study refers to time from baseline to time of measurement of the HbA1c value used in the analysis for each patient, i.e. the actual period that was being analysed. Participants who completed at least one theme on the tool were included in the analyses and compared to matched controls. Only study participants and matched controls with no reported changes to glucose-lowering medication during the follow-up period were used. The change of HbA1c was compared between study participants and matched controls (1:1 ratio) by a two-sided independent $t$ test. We needed 142 participants using the tool and 142 matched controls to have $80 \%$ power at alpha $=0.05$ to detect a significant difference between the groups, assuming that the true treatment effect of the tool is $5 \mathrm{mmol} / \mathrm{mol}$ with a standard deviation of $6 \mathrm{mmol} / \mathrm{mol}$ for the change of HbA1c. (The standard deviation for the change of HbA1c per year was estimated from observations of patients in ANDIS). Adjustments were made for age, sex and BMI using a linear regression model with group assignment as independent variable, HbA1c as dependent variable, and age, sex and BMI as covariates. Adjustment was made for diabetic complications using presence of known complications as a binary covariate in the linear model. Moreover, comorbidities were reported by participants in conjunction with questionnaires and classified manually into different disease categories (see Table 1). Adjustment was made for comorbidities using a categorical variable for the different comorbidities as a covariate in the linear model.

\section{Secondary Endpoints}

Secondary endpoints included the change of HbA1c from baseline to end of follow-up in participants using the tool compared with study participants who did not use the tool. As another secondary analysis we specifically assessed the outcomes in individuals with HbA1c above $45 \mathrm{mmol} / \mathrm{mol}$ by comparing users of the tool with matched controls as well as users of the tool with participants not using the tool. These analyses were done by two-sided independent Student's $t$ test.

The change of SF-12 scores from baseline to 3 months was analysed between randomized controls and participants with immediate access to the tool by a two-sided independent $t$ test. The intraindividual change of SF-12 scores from baseline to 6 months was analysed by paired two-sided $t$ tests (no SF-12 data were available from the matched controls).

Summary statistics are generally presented as point estimates with $95 \%$ CI. The widths of the intervals have not been adjusted for multiplicity. Statistical analyses were performed using IBM SPSS (v26). 
Table 1 Baseline characteristics of participants with T2D in randomization groups

\begin{tabular}{|c|c|c|}
\hline Characteristic & Tool $(N=112)$ & Usual care $(N=27)$ \\
\hline Female sex (\%) & 44 & 32 \\
\hline Age (years) & $63(9)$ & $65(11)$ \\
\hline Diabetes duration (years) & $3.1(1.9)$ & $3.3(2.0)$ \\
\hline Body mass index ${ }^{\mathrm{a}}\left(\mathrm{kg} / \mathrm{m}^{2}\right)$ & $30(6)$ & $28(4)$ \\
\hline Glycated haemoglobin level $(\mathrm{mmol} / \mathrm{mol})$ & $45(8)$ & $43(6)$ \\
\hline \multicolumn{3}{|l|}{ Glucose-lowering medication (\%) } \\
\hline None & 20 & 19 \\
\hline Oral only & 76 & 73 \\
\hline Oral and insulin & 2 & 4 \\
\hline Insulin only & 1 & 3 \\
\hline Known diabetic complications $(\%)^{\mathrm{b}}$ & 10 & 4 \\
\hline \multicolumn{3}{|l|}{ Reported comorbidities $(\%)^{c}$} \\
\hline Psychiatric & 0 & 0 \\
\hline Reaction to severe stress leading to sick leave & 0 & 2 \\
\hline Orthopaedic & 7 & 5 \\
\hline Gastrointestinal and hepatic & 0 & 0 \\
\hline Arrythmias & 1 & 3 \\
\hline Cardiovascular disease (incl. myocardial infarction, stroke, severe heart failure) & 2 & 8 \\
\hline Cancer & 1 & 0 \\
\hline Pulmonary & 1 & 0 \\
\hline Neurological & 0 & 0 \\
\hline Rheumatological & 0 & 0 \\
\hline Thyroid & 1 & 0 \\
\hline
\end{tabular}

Data are $n$ (\%) or mean (SD). Percentages may not total 100 because of rounding

${ }^{a}$ The body mass index is the weight in kilograms divided by the square of the height in metres

${ }^{b}$ Include known retinopathy, neuropathy, nephropathy or diabetic foot disease

${ }^{\mathrm{c} C o m o r b i d i t i e s ~ w e r e ~ r e p o r t e d ~ b y ~ p a r t i c i p a n t s ~ i n ~ c o n j u n c t i o n ~ w i t h ~ q u e s t i o n n a i r e s, ~ c l a s s i f i e d ~ m a n u a l l y ~ i n t o ~ d i f f e r e n t ~ d i s e a s e ~}$ categories and presented as presence in \% of cases

\section{RESULTS}

First, we assessed the effects on HbA1c from baseline to 12 weeks between randomization groups. Second, the changes of HbA1c from baseline to end of follow-up were compared between users of the tool and matched controls. Third, the effects on quality of life were investigated in both individuals with and without diabetes. 


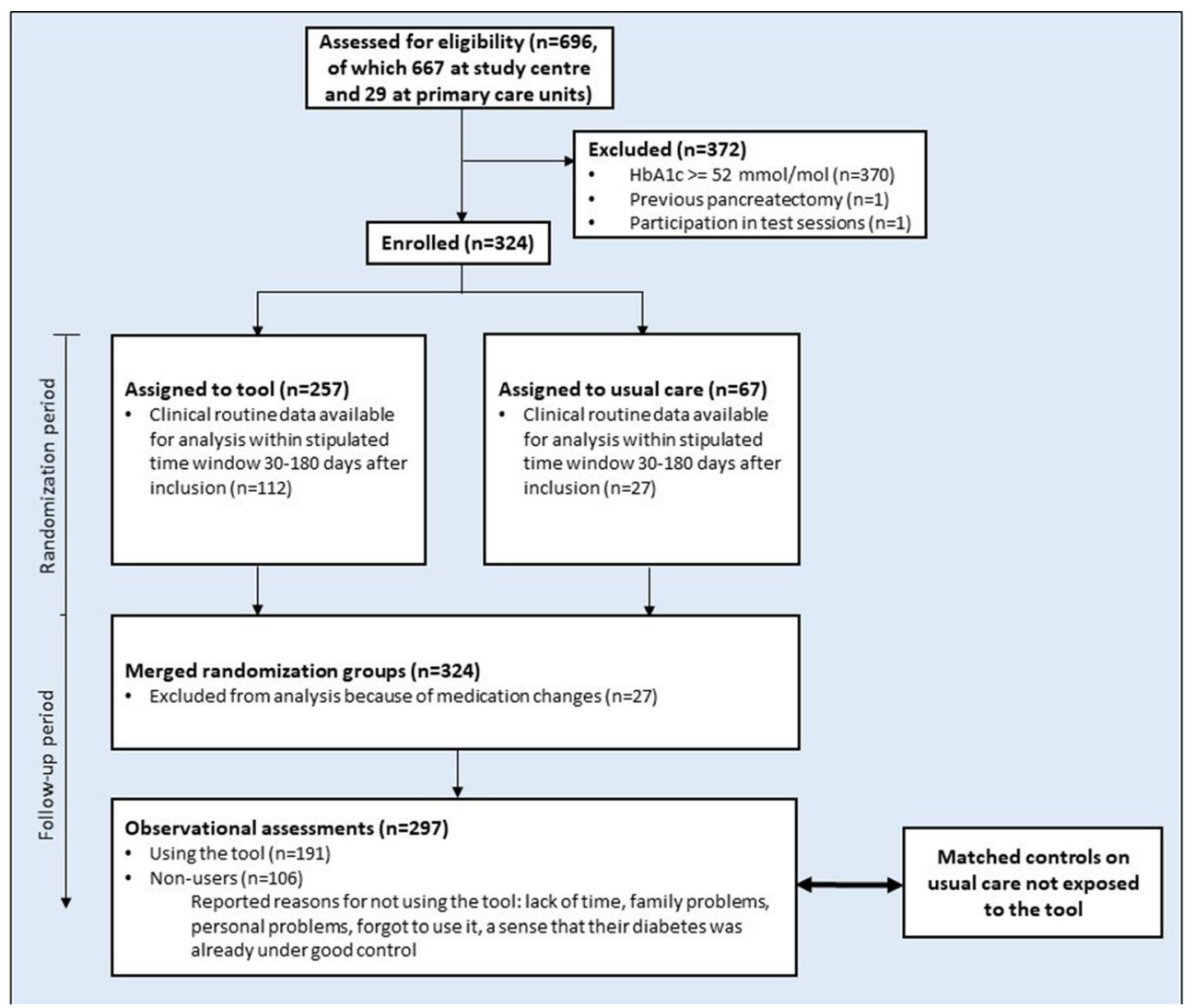

Fig. 1 Study profile as CONSORT diagram for assessments of glucose control. A total of 324 individuals were randomized to usual care or to access the tool (ratio 1:4). Comparisons between randomized controls and participants with immediate access to the tool were performed for participants with T2D in the Scania region who had an HbAlc measurement obtained in routine care within 30-180 days after study inclusion, utilizing the measurement nearest to 90 days after inclusion. Participants were included in this analysis independent of frequency of using the tool. After 12 weeks, the randomization groups were merged to enable all participants to use the tool during a

\section{Glucose Control in Participants with T2D Randomized to the Tool or Usual Care for 12 Weeks}

A total of 667 individuals with T2D attended screening visits for analysis of HbA1c. Of those, 295 had HbA1c below $52 \mathrm{mmol} / \mathrm{mol}$ and were enrolled. Another 29 individuals were included after HbA1c screening at their primary care unit because of inability to attend screening visits at the study centre (Fig. 1). follow-up period of 359 days on average. For analysis from baseline to end of follow-up, the baseline $\mathrm{HbAlc}$ at inclusion and the $\mathrm{HbAlc}$ measurement obtained in clinical care nearest to 365 days after inclusion within a window of 18 months after inclusion were used. Participants who completed at least one theme on the tool were included in the analyses and compared to matched controls. Only study participants and matched controls with no reported changes to glucose-lowering medication during the follow-up period were used

We aimed to evaluate the intervention in a setting that was as similar as possible to ordinary clinical conditions by observing the metabolic outcomes in participants with T2D who had access to the tool in addition to usual care.

The existence of effect was investigated by randomizing the participants to access the tool immediately or wait for 12 weeks (ratio 4:1). Follow-up data from routine clinical measurements were available for 139 participants within 
the stipulated time window (average 100 days between inclusion and follow-up measurement [interquartile range 65-134]; Table 1). The average $\mathrm{HbA} 1 \mathrm{c}$ decreased by $1 \mathrm{mmol} / \mathrm{mol}$ relative to baseline (95\% CI -2 to $0 ; n=112)$ in participants with access to the tool. In participants randomized to wait, average HbA1c increased by $2 \mathrm{mmol} / \mathrm{mol}$ relative to baseline (95\% CI $0-3 ; n=27)$. The mean difference between the groups was $-2 \mathrm{mmol} / \mathrm{mol}$ (95\% CI -4 to $0 ; P=0.02$ ).

\section{Glucose Control from Baseline to End of Follow-up in Users of the Tool Compared with Controls}

We also aimed to assess more long-term outcomes. The randomization groups were merged after the initial 12-week period to enable all patients to use the tool during an extended open-label period (Fig. 1). Twenty-seven participants had reported medication changes during the follow-up period and were therefore excluded from analysis. All remaining 297 participants had a routine clinical measurement of HbA1c within 18 months after inclusion; the value nearest to 1 year after inclusion in the study was used for comparisons. The average time between inclusion and final HbA1c measurement was 359 days (interquartile range 288-432). A total of 106 participants never used the tool despite having access to it (Table 2). Reported reasons included lack of time, a sense that their diabetes was already under good control, and personal or family issues that received higher priority. Those who used the tool $(n=191)$ did on average complete 13 themes during follow-up.

\section{Comparison Between Users of the Tool and Non-users}

The change of HbA1c from baseline to end of follow-up was compared between participants who used the tool and participants who did not use it. Those who used it had a $1 \mathrm{mmol} / \mathrm{mol}$ average reduction of $\mathrm{HbA} 1 \mathrm{c}(95 \% \mathrm{CI}-1.4$ to
$0.4 ; n=191)$, while HbA1c increased by $2 \mathrm{~mol} / \mathrm{mol}$ on average in non-users $(95 \% \mathrm{CI}$ $0.7-2.7 ; n=106)$. The mean difference between the groups was $-2 \mathrm{mmol} / \mathrm{mol}(95 \% \mathrm{CI}-4$ to $-1 ; P=0.02 ; P=0.001$ after adjusting for age, sex and BMI; $P=0.001$ after adjusting for diabetic complications and $P=0.002$ after adjusting for comorbidities).

\section{Comparison Between Users of the Tool and Matched Controls}

The users of the tool were also compared with matched controls (Table 3). Average HbA1c increased by $1 \mathrm{mmol} / \mathrm{mol}$ in the matched controls over a similar time period (95\% CI 0-2), and the mean difference between users of the tool and matched controls was $-2 \mathrm{mmol} / \mathrm{mol}$ (95\% CI -3 to $0 ; P=0.005 ; P=0.004$ after adjusting for age, sex and BMI).

\section{Effect in Participants with Higher Baseline HbA1c}

The study participants had a broad range of HbA1c (interquartile range $39-47 \mathrm{mmol} / \mathrm{mol}$ ). In order to assess the effect of the tool in patients with less optimal glucose control, we specifically studied those with HbA1c above $45 \mathrm{mmol} / \mathrm{mol}$ as a secondary analysis. Users of the tool with $\mathrm{HbA} 1 \mathrm{c}$ above $45 \mathrm{mmol} / \mathrm{mol}$ at baseline demonstrated an $\mathrm{HbA1c}$ reduction of $3 \mathrm{mmol} / \mathrm{mol}$ during follow-up (95\% CI -5 to $-1)$. The corresponding change in non-users was $1 \mathrm{mmol} / \mathrm{mol}$ (95\% CI -2 to 3 ). The mean difference between the groups was $-4 \mathrm{mmol} / \mathrm{mol}(95 \% \mathrm{CI}-7$ to $0 ; P=0.03)$. This was paralleled by a $-4 \mathrm{mmol} / \mathrm{mol}$ mean difference between users of the tool and matched controls at HbA1c above $45 \mathrm{mmol} / \mathrm{mol}$ (95\% CI -7 to $-2 ; P=0.003)$. Overall, there was an association between high baseline $\mathrm{HbA1c}$ and larger HbA1c reduction in users of the tool $(P<0.001$ using linear regression $)$ but not in matched controls $(P=0.7 ; \quad P<0.001$ when exposure to the tool and baseline HbA1c were evaluated as an interaction term in the linear model). 
Table 2 Baseline characteristics of participants with T2D in long-term follow-up

\begin{tabular}{|c|c|c|c|}
\hline Characteristic & $\begin{array}{l}\text { Users } \\
(N=191)\end{array}$ & $\begin{array}{l}\text { Non-users } \\
(N=106)\end{array}$ & $\begin{array}{l}\text { All } \\
(N=297)\end{array}$ \\
\hline Female sex (\%) & 44 & 38 & 42 \\
\hline Age (years) & $63(9)$ & $62(10)$ & $63(10)$ \\
\hline Diabetes duration (years) & $3.0(1.9)$ & $3.1(1.7)$ & $3.0(1.8)$ \\
\hline Body mass index ${ }^{a}\left(\mathrm{~kg} / \mathrm{m}^{2}\right)$ & $29(6)$ & $30(6)$ & $29(6)$ \\
\hline Glycated haemoglobin level $(\mathrm{mmol} / \mathrm{mol})$ & $43(6)$ & $44(7)$ & $44(7)$ \\
\hline \multicolumn{4}{|l|}{ Glucose-lowering medication (\%) } \\
\hline None & 22 & 29 & 24 \\
\hline Oral only & 75 & 68 & 73 \\
\hline Oral and insulin & 1 & 1 & 1 \\
\hline Insulin only & 1 & 0 & 1 \\
\hline Known diabetic complications $(\%)^{\mathrm{b}}$ & 5 & 8 & 6 \\
\hline \multicolumn{4}{|l|}{ Reported comorbidities $(\%)^{c}$} \\
\hline Psychiatric & 1 & 0 & 1 \\
\hline Reaction to severe stress leading to sick leave & 1 & 1 & 1 \\
\hline Orthopaedic & 8 & 7 & 7 \\
\hline Gastrointestinal and hepatic & 0 & 1 & 1 \\
\hline Arrythmias & 2 & 1 & 2 \\
\hline $\begin{array}{l}\text { Cardiovascular disease (incl. myocardial infarction, stroke, severe } \\
\text { heart failure) }\end{array}$ & 3 & 3 & 3 \\
\hline Cancer & 1 & 1 & 1 \\
\hline Pulmonary & 1 & 1 & 1 \\
\hline Neurological & 0 & 0 & 0 \\
\hline Rheumatological & 0 & 0 & 0 \\
\hline Thyroid & 1 & 0 & 1 \\
\hline
\end{tabular}

Data are $n$ (\%) or mean (SD). Percentages may not total 100 because of rounding

${ }^{a}$ The body mass index is the weight in kilograms divided by the square of the height in metres

b Include known retinopathy, neuropathy, nephropathy or diabetic foot disease

${ }^{\mathrm{c}}$ Comorbidities were reported by participants in conjunction with questionnaires, classified manually into different disease categories and presented as presence in $\%$ of cases 
Table 3 Baseline characteristics of users of the tool and matched controls with T2D

\begin{tabular}{lll}
\hline Characteristic & Users $(\boldsymbol{N}=\mathbf{1 9 1})$ & Matched controls $(\boldsymbol{N}=\mathbf{1 9 1})$ \\
\hline Female sex $(\%)$ & 44 & 44 \\
Age (years) & $63(9)$ & $63(10)$ \\
Body mass index ${ }^{\mathrm{a}}\left(\mathrm{kg} / \mathrm{m}^{2}\right)$ & $29(6)$ & $29(5)$ \\
Glycated haemoglobin level $(\mathrm{mmol} / \mathrm{mol})$ & $43(6)$ & $42(5)$ \\
\hline
\end{tabular}

Data are $n(\%)$ or mean (SD)

${ }^{a}$ The body mass index is the weight in kilograms divided by the square of the height in metres

\section{Assessment of Quality of Life in Both Individuals with Diabetes and Those Without Diabetes}

There is currently a lack of knowledge of the effects of digital interventions on quality of life $[2,5]$. In addition to studying the effects of the tool on metabolic control, we therefore investigated its influence on quality of life. For these analyses, participants from all over Sweden were enrolled, also individuals without diabetes $(n=1914$ in total, of which 880 had T2D; Table 4). The SF-12 questionnaire was completed at baseline and after the 3-month randomization period as well as 6 months after accessing the tool. The questionnaire measures perceived general health, feelings and ability to perform daily activities. The individual components are subsequently combined to an overall physical health score and a mental health score [25].

Perceived general health was improved at 3 months relative to baseline in those randomized to access the tool $(n=1512)$ compared with randomized controls $(n=402$; mean difference 0.08 a.u. on a 5-point Likert scale [95\% CI $0.01-0.16] ; P=0.045)$. The mean difference in overall SF-12 scores between the groups did not reach statistical significance at 3 months.

Next, we analysed the intraindividual change in participants between baseline and 6 months of access to the tool and observed a statistically significant improvement in both the overall physical health score $(P<0.001)$ and mental health score $(P=0.006$; data from baseline as well as 6 months were available from 1149 participants, of which 530 had T2D). This was accompanied by improvements in the components of SF-12 that reflect perceived general health, energy, disturbance by pain, and the ability to perform work or other daily tasks despite physical or mental distress.

It is of note that the participants without diabetes reported better physical health score at baseline compared with participants with T2D but poorer mental health score (Table 4 ). The improvements of overall health scores from baseline to 6 months were mainly observed in individuals without diabetes. Perceived general health and the ability to perform work or other daily activities despite physical problems were the components that reached statistically significant improvements in participants with T2D.

\section{DISCUSSION}

This study demonstrated improved HbA1c in participants with T2D using the new digital tool. The improvements were observed during a 12 -week randomization period relative to randomized wait list controls and during a subsequent follow-up period of 1 year on average when users of the tool were compared with both non-users and matched controls, respectively.

The tool adds a new facet to lifestyle management by its emphasis on self-reflection. A major asset is also the ability to utilize the tool in healthcare as a complement to usual care without the need for additional personnel resources.

All HbA1c measures during follow-up were obtained in routine clinical care rather than at 
Table 4 Baseline characteristics of participants in quality of life assessments

\begin{tabular}{|c|c|c|c|}
\hline Characteristic & $\begin{array}{l}\text { With T2D } \\
(N=880)\end{array}$ & $\begin{array}{l}\text { Without T2D } \\
(N=1034)\end{array}$ & $\begin{array}{l}\text { All } \\
(N=1914)\end{array}$ \\
\hline Female sex $(\%)$ & 48 & 90 & 71 \\
\hline Age (years) & $61(10)$ & $47(9)$ & $54(12)$ \\
\hline Body mass index ${ }^{\mathrm{a}}\left(\mathrm{kg} / \mathrm{m}^{2}\right)$ & $30(6)$ & $27(5)$ & $28(6)$ \\
\hline Baseline physical SF-12 health score ${ }^{\mathrm{b}}$ & $41.6(8.3)$ & $43.8(7.7)$ & $42.8(8.0)$ \\
\hline Baseline mental SF-12 health score & $48.1(10.7)$ & $39.5(11.5)$ & $43.4(11.9)$ \\
\hline \multicolumn{4}{|l|}{ Reported comorbidities ${ }^{c}-$ no. of cases } \\
\hline Psychiatric & 7 & 13 & 20 \\
\hline Reaction to severe stress leading to sick leave & 11 & 48 & 59 \\
\hline Orthopaedic & 33 & 26 & 59 \\
\hline Gastrointestinal and hepatic & 4 & 3 & 7 \\
\hline Arrythmias & 4 & 3 & 7 \\
\hline $\begin{array}{l}\text { Cardiovascular disease (incl. myocardial infarction, stroke, severe } \\
\text { heart failure) }\end{array}$ & 13 & 3 & 16 \\
\hline Cancer & 2 & 2 & 4 \\
\hline Pulmonary & 3 & 2 & 5 \\
\hline Neurological & 2 & 1 & 3 \\
\hline Rheumatological & 4 & 4 & 8 \\
\hline Thyroid & 0 & 10 & 10 \\
\hline
\end{tabular}

Data are $n$ (\%) or mean (SD). Percentages may not total 100 because of rounding. The participants with T2D include both patients from the Scania region involved in glycaemic assessments and patients with T2D outside of the Scania region ${ }^{a}$ The body mass index is the weight in kilograms divided by the square of the height in metres

${ }^{b}$ The distinct components of the SF-12 survey can be summarized to a physical health score and a mental health score, with higher values implying better state

${ }^{c}$ Comorbidities were reported by participants in conjunction with questionnaires, classified manually into different disease categories and presented as number of cases

study visits. While this procedure only permits blood sampling at less distinct intervals, it is nonetheless a strength in this particular case, as it reduces the risk for influence at study visits ('placebo') and helps assess the effects of the tool on its own in a setting that is as close as possible to a real clinical context. It also enabled us to obtain long-term outcome measures from all 297 participants, including non-users, which is important since high attrition is usually a major problem in studies of digital interventions $[26,27]$.

The metabolic improvement in response to the tool was sustained during the entire followup of 1 year on average, making this one of the longest investigations of digital lifestyle treatment to date [9-11]. The study has also a number of limitations. No adjustment was made for multiple comparisons, and the variables should therefore be interpreted on the basis of the overall pattern of outcomes. A 
fundamental problem with lifestyle studies is selection bias because of variation in initial motivation between patients [28]. In particular, differences in initial motivation may influence the comparisons between participants using the tool and non-users. It is therefore important that statistically significant differences in change of glycaemic control were also observed between users of the tool and randomized controls and between users of the tool and matched controls. We adjusted for a number of potential confounders such as age, sex and BMI as well as complications and comorbidities between users and non-users, but we cannot exclude that unmeasured confounders may have affected the outcomes.

The average effect of the intervention was $2 \mathrm{mmol} / \mathrm{mol}$ compared with controls, with a pronounced effect in patients with poorer glucose control $(4 \mathrm{mmol} / \mathrm{mol}$ average reduction of HbA1c in users of the tool relative to matched controls at HbA1c above $45 \mathrm{mmol} / \mathrm{mol}$ ). The sustained improvement during 1 year in response to the tool is of interest in light of previous meta-analyses that have shown initial effects of lifestyle programmes that decline after 6 months [9-11]. In a Cochrane report, the average $\mathrm{HbA1c}$ reduction in response to digital interventions with follow-up time ranging from 1 to $12 \mathrm{months}$ was $2.3 \mathrm{mmol} / \mathrm{mol}$, with effects observed predominantly in patients with HbA1c above $52 \mathrm{mmol} / \mathrm{mol}$ [5]. It is also of note that a recent traditional behavioural intervention programme with group-based sessions in individuals with HbA1c between 42 and $47 \mathrm{mmol} / \mathrm{mol}$ led to a $1.3 \mathrm{mmol} / \mathrm{mol}$ reduction of HbA1c [29]. Moreover, it may be of relevance to compare our results with a recent pharmacological intervention study, which showed a $2 \mathrm{mmol} / \mathrm{mol}$ reduction of HbA1c over 6 months in response to sulfonylureas, dipeptidyl peptidase 4 inhibitors or sodium-glucose transporter 2 inhibitors added to metformin in patients with relatively well-controlled diabetes (i.e. similar to our cohort) [30]. This indicates that the glycaemic effect of using the tool in this patient group appears to be on par with common antihyperglycemic drugs.

A $2 \mathrm{mmol} / \mathrm{mol}$ average $\mathrm{HbA} 1 \mathrm{c}$ reduction in individuals with baseline HbA1c below
$52 \mathrm{mmol} / \mathrm{mol}$, as observed in response to the tool, would according to an estimation from the Norfolk cohort result in $10 \%$ reduction of diabetes-attributable death in the population [31]. Furthermore, it is well established that early glucose-lowering interventions may reduce beta-cell load and prevent beta-cell exhaustion, thereby reducing the risk for subsequent loss of glycaemic control [32]. Interestingly, it was shown in the VERIFY study that treatment failure (HbA1c $>52 \mathrm{mmol} / \mathrm{mol}$ ) occurred in $44 \%$ of patients with combination therapy and $62 \%$ of those with metformin monotherapy within a 5 -year period [33]. The digital tool could therefore offer a new low-cost means to further reduce glucose levels and help prevent or delay glycaemic dysregulation.

As a secondary analysis, the tool was shown to improve physical and mental assessments of quality of life, especially in individuals without diabetes, suggesting that it may be useful to broader population groups in addition to managing metabolic health. It is of note that the initial mental health score was significantly lower in the individuals without diabetes than in participants with T2D, possibly because individuals without diabetes who wanted to take part in the study had needs in other health domains, as suggested by the higher numbers of severe stress reactions reported in those participants (Table 4). This could potentially explain the larger improvement in quality of life that was observed in the participants without diabetes.

In order for digital lifestyle solutions to be scalable and broadly applicable, it is essential that efficacy does not depend on in-person reinforcement or increased healthcare activities $[9,34]$. We therefore provided the tool as a stand-alone support under conditions that were as similar as possible to routine clinical conditions. While this may reduce adherence compared with stricter regimes, it increases the general significance of the results by demonstrating what can be expected in real-life situations over extended time. Approximately 36\% (106 of the 297 participants) did not use the tool during the long-term follow-up. These rates are comparable with what has previously been observed in meta-analyses of digital tools 
$[26,27]$. We found, however, a significant difference in glycaemic change between randomization groups (when participants were included independent of use), suggesting that the tool has broad utility even when adherence is variable. The tool is provided for free to the individual patient via academic institutions, and the marginal cost is fundamentally lower than pharmacological treatment and existing lifestyle intervention programmes. Clinical applicability is consequently much less sensitive to variation in compliance. The tool may also release resources for other forms of lifestyle support to patients for whom digital solutions are not relevant [21].

\section{CONCLUSIONS}

The study presents a self-reflective approach to lifestyle treatment that has previously been lacking. Patients with T2D using the tool had improved long-term metabolic control with a pronounced effect in those with poorer initial glucose control. The tool also improved both physical and mental aspects of quality of life, in particular in individuals without diabetes. In view of the severe clinical need for scalable patient-centric solutions, the findings offer a new means for affordable lifestyle management with sustained efficacy that could benefit a large number of patients.

\section{ACKNOWLEDGEMENTS}

We thank Svetlana Johansson, Maria Fälemark, Helene Ferm, Louise Qvist and Jessica Hedin for managing screening visits. We also thank the patients and colleagues who participated in the development and evaluation of the tool. Patients with T2D were involved in developing the tool and provided continuous feedback on language, style, design and relevance of content.

Funding. The study and the journal's Rapid Service Fee was supported by grants from the
Knut and Alice Wallenberg Foundation and the Ragnar Söderberg Foundation.

Authorship. All named authors meet the International Committee of Medical Journal Editors criteria for authorship for this article, take responsibility for the integrity of the work as a whole, and have given their approval for this version to be published.

Author Contributions. CD analysed data and co-wrote the manuscript. BA was involved in designing the tool and the study, analysed data and coordinated the clinical study. AHR conceived the project, designed the tool, analysed data, was the principal investigator of the study and co-wrote the manuscript.

Disclosures. Chinmay Dwibedi, Birgitta Abrahamsson and Anders H. Rosengren have nothing to disclose.

Compliance with Ethics Guidelines. The study procedures were approved by the regional ethics review committee of Gothenburg, Sweden; no. 651/16, approved 22 August 2016, and subsequent amendments. The study was performed according to the Declaration of Helsinki and its later amendments. All participants provided written informed consent to participate in the study.

Data Availability. The datasets generated during and/or analyzed during the current study are available from the corresponding author on reasonable request.

Open Access. This article is licensed under a Creative Commons Attribution-NonCommercial 4.0 International License, which permits any non-commercial use, sharing, adaptation, distribution and reproduction in any medium or format, as long as you give appropriate credit to the original author(s) and the source, provide a link to the Creative Commons licence, and indicate if changes were made. The images or other third party material in this article are included in the article's Creative Commons licence, unless indicated otherwise in a credit line to the material. If material is not included 
in the article's Creative Commons licence and your intended use is not permitted by statutory regulation or exceeds the permitted use, you will need to obtain permission directly from the copyright holder. To view a copy of this licence, visit http://creativecommons.org/licenses/by$\mathrm{nc} / 4.0 /$.

\section{REFERENCES}

1. Davies MJ, D'Alessio DA, Fradkin J, et al. Management of hyperglycaemia in type 2 diabetes, 2018. A consensus report by the American Diabetes Association (ADA) and the European Association for the Study of Diabetes (EASD). Diabetologia. 2018;61(12):2461-98.

2. Chatterjee S, Davies MJ, Heller S, Speight J, Snoek FJ, Khunti K. Diabetes structured self-management education programmes: a narrative review and current innovations. Lancet Diabetes Endocrinol. 2018;6(2):130-42.

3. Peyrot M, Burns KK, Davies M, et al. Diabetes Attitudes Wishes and Needs 2 (DAWN2): a multinational, multi-stakeholder study of psychosocial issues in diabetes and person-centred diabetes care. Diabetes Res Clin Pract. 2013;99(2):174-84.

4. Nordyke RJ, Appelbaum K, Berman MA. Estimating the impact of novel digital therapeutics in type 2 diabetes and hypertension: health economic analysis. J Med Internet Res. 2019;21(10):e15814.

5. Pal K, Eastwood SV, Michie S, et al. Computer-based diabetes self-management interventions for adults with type 2 diabetes mellitus. Cochrane Database Syst Rev. 2013;(3):CD008776.

6. Faruque LI, Wiebe N, Ehteshami-Afshar A, et al. Effect of telemedicine on glycated hemoglobin in diabetes: a systematic review and meta-analysis of randomized trials. Can Med Assoc J. 2017;189(9): E341-64.

7. Kebede MM, Zeeb H, Peters M, Heise TL, Pischke CR. Effectiveness of digital interventions for improving glycemic control in persons with poorly controlled type 2 diabetes: a systematic review, meta-analysis, and meta-regression analysis. Diabetes Technol Ther. 2018;20(11):767-82.

8. Shan R, Sarkar S, Martin SS. Digital health technology and mobile devices for the management of diabetes mellitus: state of the art. Diabetologia. 2019;62(6):877-87.
9. Bendig E, Bauereiss N, Ebert DD, Snoek F, Andersson $\mathrm{G}$, Baumeister $\mathrm{H}$. Internet-based interventions in chronic somatic disease. Deutsch Arzteblatt Int. 2018;115(40):659-65.

10. Kitsiou S, Pare G, Jaana M, Gerber B. Effectiveness of mHealth interventions for patients with diabetes: an overview of systematic reviews. PLoS One. 2017;12(3):e0173160.

11. Greenwood DA, Gee PM, Fatkin KJ, Peeples M. A systematic review of reviews evaluating technologyenabled diabetes self-management education and support. J Diabetes Sci Technol. 2017;11(5): 1015-27.

12. Lie SS, Karlsen B, Niemiec CP, et al. Written reflection in an eHealth intervention for adults with type 2 diabetes mellitus: a qualitative study. Patient Prefer Adherence. 2018;12:311-20.

13. Lakerveld J, Palmeira AL, van Duinkerken E, et al. Motivation: key to a healthy lifestyle in people with diabetes? Current and emerging knowledge and applications. Diabet Med. 2020;37(3):464-72.

14. Steele CM. The psychology of self-affirmation: Sustaining the integrity of the self. Adv Exp Soc Psychol. 1988;21:261-302.

15. Epton T, Harris PR. Self-affirmation promotes health behavior change. Health Psychol. 2008;27: 746-52.

16. Cohen GL, Sherman DK. The psychology of change: self-affirmation and social psychological intervention. Annu Rev Psychol. 2014;65:333-71.

17. Falk EB, O'Donnell MB, Cascio CN, et al. Self-affirmation alters the brain's response to health messages and subsequent behavior change. Proc Natl Acad Sci USA. 2015;112:1977-82.

18. Miller WR, Rollnick ST. Things that motivational interviewing is not. Behav Cogn Psychother. 2009;37:129-40.

19. Ameli M, Dattilio FM. Enhancing cognitive behavior therapy with logotherapy: techniques for clinical practice. Psychotherapy. 2013;50:387-439.

20. Michie S, Ashford S, Sniehotta FF, Dombrowski SU, Bishop A, French DP. A refined taxonomy of behaviour change techniques to help people change their physical activity and healthy eating behaviours: the CALO-RE taxonomy. Psychol Health. 2011;26:1479-98.

21. Powers MA, Bardsley JK, Cypress M, et al. Diabetes self-management education and support in adults with type 2 diabetes: a consensus report of the American Diabetes Association, the Association of 
Diabetes Care \& Education Specialists, the Academy of Nutrition and Dietetics, the American Academy of Family Physicians, the American Academy of PAs, the American Association of Nurse Practitioners, and the American Pharmacists Association. Diabetes Care. 2020;43(7):1636-49.

22. WHOQOL-SRPB Field-Test Instrument. Department of Mental Health and Substance Dependence. WHO, Geneva; 2002. http://www.who.int/mental_ health/media/en/622.pdf. Accessed 27 Dec 2021

23. WHOQOL-SRPB Users Manual, Scoring and Coding for the WHOQOL SRPB Field-Test Instrument. Department of Mental Health and Substance Dependence, World Health Organization, Geneva; 2002. http://www.who.int/mental_health/media/ en/620.pdf. Accessed 27 Dec 2021

24. WHOQoL SRPB Group. A cross-cultural study of spirituality, religion, and personal beliefs as components of quality of life. Soc Sci Med. 2006;62: 1486-97.

25. Jenkinson C, Layte R, Jenkinson D, et al. A shorter form health survey: can the SF-12 replicate results from the SF-36 in longitudinal studies? J Public Health Med. 1997;19(2):179-86.

26. Kelders SM, Kok RN, Ossebaard HC, Van GemertPijnen JE. Persuasive system design does matter: a systematic review of adherence to web-based interventions. J Med Internet Res. 2012;14(6):e152.

27. Meyerowitz-Katz G, Ravi S, Arnolda L, et al. Rates of attrition and dropout in app-based interventions for chronic disease: systematic review and metaanalysis. J Med Internet Res. 2020;22(9):e20283.
28. Mohr DC, Spring B, Freedland KE, et al. The selection and design of control conditions for randomized controlled trials of psychological interventions. Psychother Psychosom. 2009;78(5): 275-84.

29. Valabhji J, Barron E, Bradley D, et al. Early outcomes from the English National Health Service Diabetes Prevention Programme. Diabetes Care. 2020;43:152-60.

30. Wilding J, Godec T, Khunti K, et al. Changes in HbA1c and weight, and treatment persistence, over the 18 months following initiation of second-line therapy in patients with type 2 diabetes: results from the United Kingdom Clinical Practice Research Datalink. BMC Med. 2018;16:116.

31. Khaw KT, Wareham N, Luben R, et al. Glycated haemoglobin, diabetes, and mortality in men in Norfolk cohort of European Prospective Investigation of Cancer and Nutrition (EPIC-Norfolk). BMJ. 2001;322:15.

32. White MG, Shaw JA, Taylor R. Type 2 diabetes: the pathologic basis of reversible $\beta$-cell dysfunction. Diabetes Care. 2016;39(11):2080-8.

33. Matthews DR, Paldánius PM, Proot P, et al. Glycaemic durability of an early combination therapy with vildagliptin and metformin versus sequential metformin monotherapy in newly diagnosed type 2 diabetes (VERIFY): a 5-year, multicentre, randomised, double-blind trial. Lancet. 2019;394: 1519-29.

34. Chatterjee S, Khunti K, Davies MJ. Type 2 diabetes. Lancet. 2017;389:2239-51. 